Kardiologe 2022 · 16:6-20

https://doi.org/10.1007/s12181-021-00522-4

Angenommen: 24. November 2021

Online publiziert: 6. Dezember 2021

(c) Deutsche Gesellschaft für Kardiologie - Herzund Kreislaufforschung e.V. Published by Springer Medizin Verlag $\mathrm{GmbH}$, ein Teil von Springer Nature - all rights reserved 2021, korrigierte Publikation 2021

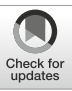

\section{Positionspapier zur Zertifizierung von Telemedizinzentren}

\author{
Empfehlungen der AG 33 Telemonitoring in der Deutschen \\ Gesellschaft für Kardiologie - Herz- und Kreislaufforschung \\ e.V. (DGK)
}

Thomas M. Helms ${ }^{1,2,13}$ für AG 33 Telemonitoring in der DGK · Christian A. Perings ${ }^{3}$. Philipp Sommer ${ }^{4}$ Friedrich Köhler ${ }^{5}$ Norbert Frey ${ }^{6} \cdot$ Stephan von Haehling ${ }^{7,8}$. Christiane Tiefenbacher ${ }^{9} \cdot$ Karin Rybak $^{10} \cdot$ Stefan Sack ${ }^{11} \cdot$ Martin Stockburger ${ }^{12}$

${ }^{1}$ Peri Cor Arbeitsgruppe Kardiologie/Ass. UCSF, Hamburg, Deutschland; ${ }^{2}$ Deutsche Stiftung für chronisch Kranke, Fürth, Deutschland; ${ }^{3}$ Kath. Klinikum Lünen-Werne, Med. Klinik I, Kardiologie, Pneumologie und Intensivmedizin, St.-Marien-Hospital Lünen, Lünen, Deutschland; ${ }^{4}$ Klinik für Elektrophysiologie und Rhythmologie, Herz- und Diabeteszentrum NRW, Bad Oeynhausen, Deutschland; ${ }^{5} \mathrm{CC} 11$, Arbeitsbereich kardiovaskuläre Telemedizin, Medizinische Klinik m. S. Kardiologie und Angiologie, CharitéUniversitätsmedizin Berlin, Berlin, Deutschland; ${ }^{6}$ Klinik für Kardiologie, Angiologie, Pneumologie, Universitätsklinikum Heidelberg, Heidelberg, Deutschland; ${ }^{7}$ Klinik für Kardiologie und Pneumologie, Herzzentrum, Universitätsmedizin Göttingen, Göttingen, Deutschland; ${ }^{8}$ Standort Göttingen, Deutsches Zentrum für Herz- und Kreislaufforschung, Göttingen, Deutschland; ${ }^{9}$ Klinik für Kardiologie, Angiologie und Pneumologie, Marien-Hospital Wesel, Wesel, Deutschland; ${ }^{10}$ Innere Medizin und Angiologie, Praxis für Kardiologie, Dessau-Roßlau, Deutschland; "Klinik für Kardiologie, Pneumologie und Internistische Intensivmedizin, München Klinik Neuperlach, München, Deutschland; ${ }^{12}$ Medizinische Klinik Nauen, Schwerpunkt Kardiologie, Havelland Kliniken, Nauen., Deutschland; ${ }^{13}$ Research, Peri Cor Arbeitsgruppe Kardiologie, Hamburg, Deutschland
Der Verlag veröffentlicht die Beiträge in der von den Autor*innen gewählten Genderform. Die Verwendung einer angemessenen gendergerechten Sprache, um Menschen in ihrer Vielfalt wertschätzend anzusprechen, wird begrüßt.

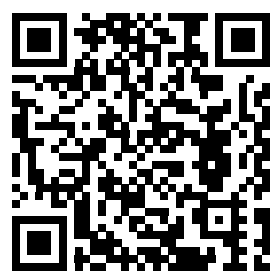

QR-Code scannen \&Beitrag online lesen

\title{
Zusammenfassung
}

Kardiovaskuläre Erkrankungen, v. a. die chronische Herzinsuffizienz, gehen mit einer erhöhten Morbidität und Mortalität einher. Im Rahmen der Patientenversorgung zeigen telemedizinische Dienstleistungen ein großes Potenzial, dem wachsenden Ungleichgewicht zwischen Behandlungsbedarf und aktuellem Versorgungsangebot entgegenzuwirken. Kardiologische Telemedizinzentren (TMZ) können somit eine flächendeckende und zeitnahe Patientenversorgung unterstützen. Zur Sicherstellung einheitlicher Prozess- und Qualitätsstandards ist die Implementierung eines systematischen Qualitätsmanagementsystems in TMZs essenziell. Die vorab definierten überprüfbaren und nachvollziehbaren Qualitätskriterien eines TMZ sollen sicherzustellen, dass die strukturierte Umsetzung von Telemonitoring und hierauf basierte strukturierte Behandlungsinterventionen eine tatsächliche Verbesserung der Versorgung herzkranker Patienten bewirken können. Zudem gewährleistet die Zertifizierung des Qualitätsmanagementsystems, dass die Strukturen und Prozesse eines TMZ an den gesetzlichen und normativen Anforderungen ausgerichtet sind.

\section{Schlüsselwörter}

Telemedizin · Telemonitoring · Qualitätsmanagement · Zertifizierung · Versorgungsqualität

\section{Präambel}

Diese Stellungnahme der Deutschen Gesellschaft für Kardiologie - Herz- und Kreislaufforschung e.V. (DGK), ordnet die aktuelle Versorgungssituation von Patienten mit kardiologischen Erkrankungen durch Telemedizinische Zentren (TMZ) in
Deutschland ein und zeigt den dringenden Handlungsbedarffür die Zertifizierung der Qualitätsmanagementsysteme in kardiologisch ausgerichteten TMZ auf. Das Positionspapier schlägt Empfehlungen zur systematischen Umsetzung telemedizinischer Überwachung und Therapie bei Herz-Kreislauf-Patienten und abgeleitete 
Hier steht eine Anzeige.

黑 Springer 
Kriterien und Strukturen für die Zertifizierung von Telemedizinzentren vor. Es ersetzt nicht die allgemeingültigen gesetzlichen oder behördlichen Bestimmungen an ein Qualitätsmanagementsystem und an dessen Zertifizierung.

\section{Einleitung und Ziele des Positionspapiers}

Vor dem Hintergrund der Diskussionen um die Qualität und Finanzierbarkeit von Gesundheitssystemen stehen die medizinischen Leistungserbringer zunehmend vor der Herausforderung, neben bestmöglicher und effizienter Versorgung gleichzeitig eine normkonforme Umsetzung von Qualitätsstandards und eine kontinuierliche Weiterentwicklung der entsprechenden qualitätssichernden Maßnahmen, darunter der Qualitätsmanagementprozesse, zu gewährleisten. Dabei gerät die Frage in den Fokus, wie die Qualität der in der intersektoralen Organisation immer komplexer werdenden Versorgungsprozesse gesichert und kontinuierlich optimiert werden kann. Zertifizierungen, die die Übereinstimmung der Arbeitsabläufe mit anerkannten Standards und Normen für einen bestimmten Zeitraum bestätigen, sind ein entscheidender Beitrag zur Sicherung von Struktur- und Prozessqualität medizinischer Versorgung. Auch im Kontext des kürzlich durch den Gemeinsamen Bundesausschuss als Untersuchungs- und Behandlungsmethode anerkannten Telemonitorings bei Herzinsuffizienz [1] ist die

\section{Abkürzungen}

\begin{tabular}{|c|c|}
\hline$C R T-D$ & $\begin{array}{l}\text { Kardiales Resynchronisationsgerät } \\
\text { mit Defibrillator }\end{array}$ \\
\hline$D G K$ & $\begin{array}{l}\text { Deutsche Gesellschaft für Kardiolo- } \\
\text { gie - Herz- und Kreislaufforschung }\end{array}$ \\
\hline DIN & Deutsches Institut für Normung \\
\hline$D Q S$ & $\begin{array}{l}\text { Deutsche Gesellschaft zur Zertifizie- } \\
\text { rung von Managementsystemen }\end{array}$ \\
\hline DS-GVO & Datenschutz-Grundverordnung \\
\hline EKG & Elektrokardiogramm \\
\hline$I C D$ & $\begin{array}{l}\text { Implantierter Kardioverter/ } \\
\text { Defibrillator }\end{array}$ \\
\hline ISO & $\begin{array}{l}\text { Internationale Organisation für } \\
\text { Normung }\end{array}$ \\
\hline KTQ & $\begin{array}{l}\text { Kooperation für Transparenz und } \\
\text { Qualität im Gesundheitswesen }\end{array}$ \\
\hline NYHA & New York Heart Association \\
\hline$S G B$ & Sozialgesetzbuch \\
\hline SOP & Standard Operation Procedures \\
\hline$T M Z$ & Telemedizinzentren \\
\hline
\end{tabular}

Einführung eines systematischen Qualitätsmanagements für die TMZ zur Prozessoptimierung und Qualitätssicherung sowie zur Absicherung der Vergleichbarkeit der Leistungen in der telemedizinischen Versorgung sinnvoll und auch als Forderung im G-BA-Beschluss enthalten [1]. Bisher existieren keine Empfehlungen zur Zertifizierung von kardiologischen TMZ.

In diesem Positionspapier wird ein Konzept für das Qualitätsmanagement und einen zukünftigen Zertifizierungsprozess von kardiologischen TMZ vorgestellt.

\section{Definition von Telemedizin/ Telemonitoring und Herausforde- rungen für die kardiologischen Telemedizinzentren}

Telemedizin umfasst die „Interaktion zwischen Akteuren in medizinischen Institutionen untereinander und die Interaktion mit Patienten auf digitalen Kommunikationswegen zum Zweck der medizinischen Diagnostik und Therapie unter Überwindung der räumlichen [und zeitlichen] Distanz" [2]. Dabei hat die Telemedizin eine grundlegende Veränderung des Wertschöpfungsnetzwerkes bewirkt und stellt daher eine radikale Innovation dar. Telemonitoring als Teilbereich der Telemedizin beschreibt "die Überwachung des Gesundheitsstatus eines Patienten mithilfe von externen oder implantierten Sensoren" [3]. Telemonitoring zeichnet sich durch komplexe Prozessabläufe und ein stark variables Umfeld aus. Zudem geht die Markteinführung telemedizinischer Konzepte mit einer Vielzahl von Barrieren einher. Zum einen sind Standards zur technischen Interoperabilität unzureichend, oder sie fehlen ganz, zum anderen ergaben sich durch die bisher fehlende Vergütung des Telemonitorings Finanzierungs- und Investitionshürden, und auch die fehlende Zertifizierung digitaler Lösungen stellt ein Problem dar [3]. Die Entwicklung von einheitlichen technischen und qualitativen Standards ist eine wichtige Voraussetzung für die Sicherstellung der notwendigen Prozess- und sich daraus ableitenden Behandlungsqualität sowie die konsekutive erfolgreicheÜberführung von telemedizinischen Dienstleistungen in die Regelversorgung.

Die Relevanz des Telemonitorings sowie die Anforderungen an Leistungen, Qua- lität und technische Umsetzung im kardiologischen Behandlungsfeld wurden bereits im Positionspapier Telemonitoring der DGK [3] und in weiteren Übersichtsarbeiten ausführlich dargestellt $[2,4,5]$. Im vorliegenden Positionspapier werden daher die grundlegenden Merkmale und Prozesse eines kardiologischen TMZ aufgezeigt, jedoch richtet sich der Fokus auf die Notwendigkeit der Zertifizierung von Qualitätsmanagementsystemen in kardiologischen TMZ.

In Deutschland wurden unterschiedliche Telemonitoring-Ansätze entwickelt, die sich in der Art der eingesetzten Geräte (externe Sensorik, wie z.B. Blutdruckmessgerät und Körperwaage, Elektrokardiogramm (EKG), und/oder telemetriefähige implantierte Geräte, wie z.B. Ereignisrekorder, Herzschrittmacher, implantierbare Kardioverter/Defibrillatoren (ICDs) oder Implantate zur kardialen Resynchronisation [CRT-D oder CRT-P]), der Intensität des Betreuungsprogramms und der organisatorischen Verankerung der TMZ unterscheiden [6]. Grundsätzlich stellen Patienten mit den nachstehend aufgelisteten kardialen Krankheitsbildern geeignete Zielgruppen für den Einsatz von Telemonitoring-Systemen dar [3]:

- chronische Herzinsuffizienz in den Krankheitsstadien II-IV nach NYHA,

- koronare Herzkrankheit,

- durchgemachter Myokardinfarkt,

- Zustand nach Katheterintervention (Dilatation [PTCA]/ Stentimplantation; elektrophysiologische Untersuchung mit Ablation von Herzrhythmusstörungen),

- durchgemachter herzchirurgischer Eingriff (z. B. nach Herztransplantation),

- unklare Synkopen, Herzrhythmusstörungen, paroxysmale Tachykardien, Vorhofflimmern/Vorhofflattern, Vorbeugung des plötzlichen Herztodes (verursacht durch Kammertachykardien oder Kammerflimmern),

- Prävention bei Hochrisikoprofil (z. B. Diabetes, Identifizierung nach ProcamScore),

- Gerinnungsmanagement bei Risikopatienten (z. B. mit implantiertem ventrikulärem Unterstützungssystem). 


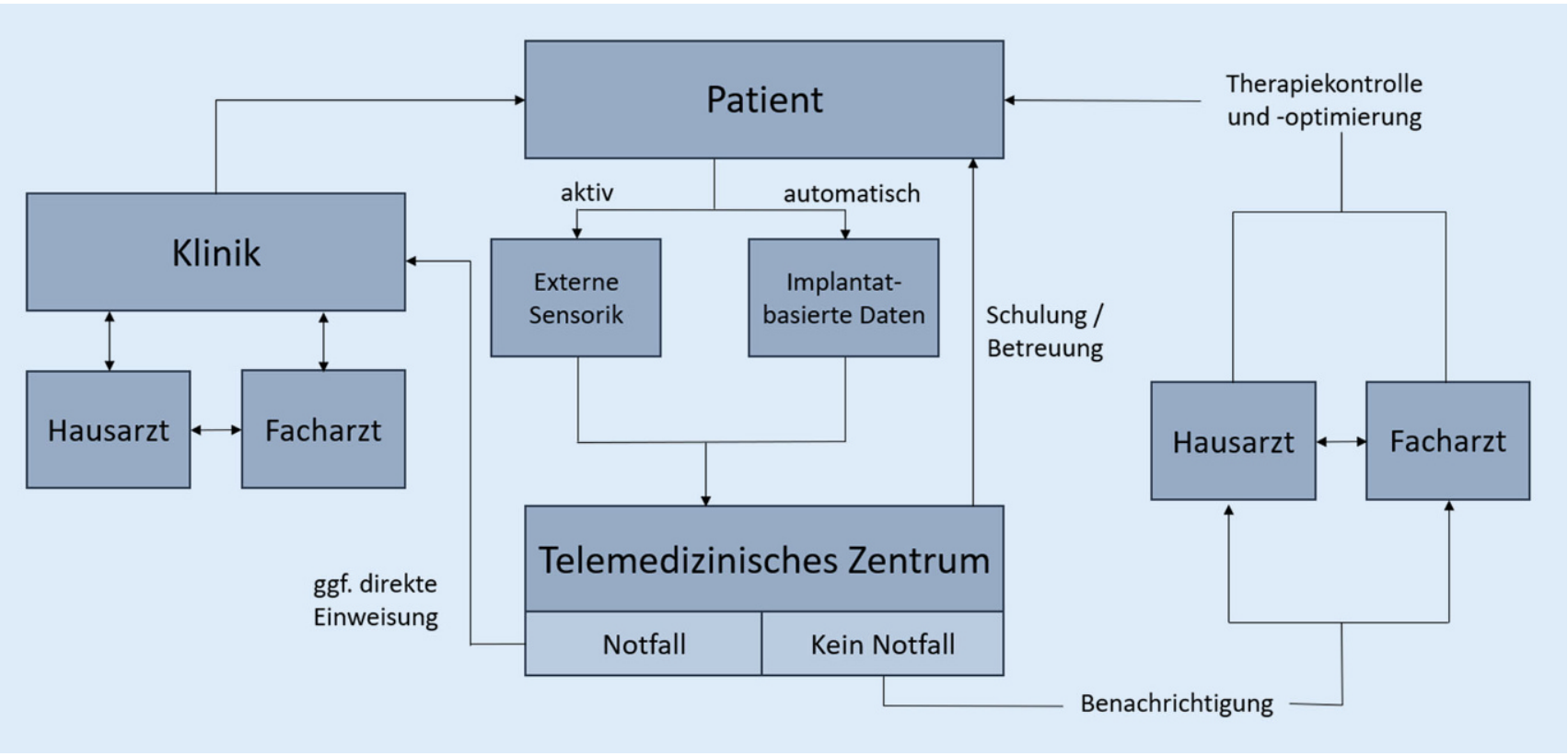

Abb. 1 ॥ Informationsprozesse bei telemedizinischer Betreuung. (Adaptiert nach [5])

Im Folgenden werden die wichtigsten telemedizinischen Informationsprozesse am Beispiel von Patienten mit chronischer Herzinsuffizienz exemplarisch durchlaufen.

\section{Informationsprozesse bei telemedizinischer Betreuung}

Da Patienten mit einer chronischen Herzinsuffizienz eine hohe Hospitalisierungsrate, eine eingeschränkte Lebensqualität sowie eine erhöhte Mortalitätsrate aufweisen [7], sind aus medizinischer Sicht die frühzeitige Erkennung einer Verschlechterung des Gesundheitszustands sowie die frühzeitige Einleitung von therapeutischen Maßnahmen erforderlich. Strukturiertes Telemonitoring mithilfe externer Sensorik und auf der Grundlage implantatbasierter Information bietet diese Möglichkeit. Physiologische Messparameter des Patienten z. B. bei externer Sensorik: Körpergewicht, Blutdruck, Herzfrequenz, EKG oder Informationen aus Herzrhythmusimplantaten, z. B. kontinuierlich erfasste Daten über körperliche Aktivität, Herzfrequenz und deren Variabilität, Herzrhythmusstörungen wie Vorhofflimmern oder Kammerarrhythmien, Thoraximpedanzverlauf zur Erkennung pulmonaler Kongestion, Herztonintensität und zusammenfassende Indizes des Herzinsuffizienzverlaufes können über
Funknetze aus dem häuslichen Umfeld in vorab definierten Zeitabständen oder in einer Akutsituation (Alarmmeldung) an ein TMZ übermittelt werden, um dort als Basis zur Veranlassung oder Einleitung individualisierter leitlinienbasierter Behandlungsinterventionen zu dienen [3]. Innerhalb des TMZ werden die übermittelten Daten des Patienten in einer elektronischen Plattform gespeichert und von medizinischem Personal mit Rückgriff auf anamnestische Daten wie Alter, Geschlecht, Begleitkrankheiten, Risikofaktoren und Medikationen analysiert. Im Rahmen des Qualitätsmanagements muss die angemessene Qualifikation des medizinischen Personals durch Ausbildung und regelmäßige spezifische Fortbildung sichergestellt werden. Bei auffälligen Befundkonstellationen werden anhand von verschriftlichten Standards mit definierter Reaktionszeit leitliniengemäße Behandlungsinterventionen abgeleitet und dem behandelnden Hausarzt oder Kardiologen oder auch dem Patienten selbst kommuniziert. Der Alarm muss dabei innerhalb von $24 \mathrm{~h}$ wahrgenommen und verarbeitet werden. Beispiele für typische Befundkonstellationen und konkrete Reaktionszeiten für den Einsatz mit Implantaten sowie externer Sensorik sind in Helms et al. [4] publiziert. Dabei arbeiten die ärztlichen und nichtärztlichen Mitarbeiter des TMZ engmaschig mit den Hausärzten bzw. Kardiologen der Patienten zusammen (s. - Abb. 1). Alarmzustände, auffällige Befundkonstellationen und abgeleitete Behandlungsinterventionen werden in einer elektronischen Plattform dokumentiert. Um eine optimale Patientenversorgung zu gewährleisten, muss das TMZ ganzjährig die fachärztliche Wahrnehmung von Alarmzuständen mit nötigenfalls angepasster Initiierung von Behandlungsinterventionen über einen 24-h-Service anbieten [5, 6].

Im Rahmen eines ganzheitlichen Betreuungskonzeptes können zusätzlich zu einem reinen Telemonitoring mit dem Patienten auch strukturierte und standardisierte Telefonate (Nurse Calls) geführt werden, die beispielsweise Informationen zur Lebensqualität, Medikation, klinischer Symptomatik und zur Häufigkeit von Arztbesuchen und Klinikaufenthalten erfassen und auffällige Befundkonstellationen des Telemonitorings ergänzen und einzuordnen helfen. Die Nurse Calls fördern zudem die Adhärenz des Patienten und können ergänzend und zusätzlich zu physiologischen Daten des Telemonitorings frühzeitig eine Verschlechterung des Gesundheitszustandes identifizieren. Darüber hinaus können Mitwirkungsmöglichkeit und Selbstwirksamkeit des Patienten („Empowerment ${ }^{\prime \prime}$ ) und seine ak- 
tivere Rolle im Versorgungsprozess durch das Angebot von Schulungen zum Thema Ernährung, Bewegung und Pharmakotherapie gestärkt werden [3]. Demnach kann ein ganzheitliches Betreuungskonzept des TMZ zusammenfassend die folgenden Elemente beinhalten [8]:

- regelmäßige qualifizierte Beobachtung und Wahrnehmung von Alarmmeldungen aus dem Telemonitoring im ambulanten Feld (ganzjähriger 24-hService),

- evidenzbasierte, leitliniengerechte Therapie durch den Hausarzt und Kardiologen, die durch den am TMZ tätigen Facharzt für Kardiologie durch befundbasierte Interventionen ergänzt wird,

- Schulung für Patienten und optional deren Angehörige zum Umgang mit der Erkrankung (Selbstwirksamkeit, Empowerment).

\section{Wirksamkeitsnachweise zum Telemonitoring}

Klare Wirksamkeitsnachweise zum erfolgreichen Einsatz von Telemonitoring unter den Bedingungen des deutschen Gesundheitswesens lieferten bereits die TIM-HF-2-Studie [9] für die Begleitung mit externer Sensorik bei Patienten mit Herzinsuffizienz und die IN-TIME-Studie [10] für das Monitoring gestützt durch Daten aus kardialen Rhythmusimplantaten. Die TIM-HF-2-Studie wies eine signifikante Verminderung von Gesamtmortalität und Hospitalisationsdauer durch die Begleitung mit hoch strukturiertem Telemonitoring im Vergleich zu konventioneller Therapie bei symptomatischen Patienten mit einer chronischen Herzinsuffizienz ohne relevante begleitende depressive Erkrankung nach [9]. Die IN-TIME-Studie erbrachte im randomisierten Vergleich mit konventionellem Patientenmanagement ebenfalls einen Vorteil im Hinblick auf Herzinsuffizienz und Sterblichkeit durch das konsequent strukturierte implantatbasierte Telemonitoring. Beiden Studien sind die konsequente Wahrnehmung der aus dem Telemonitoring verfügbaren Information und die nachfolgende, zeitnahe strukturierte und standardisierte Umsetzung von befund- und leitlinienbasierten Behandlungsinterventionen gemeinsam.
Als zielführend hat sich also nicht die reine Verfügbarkeit von telemedizinischen Daten der Patienten erwiesen, sondern das strukturierte therapeutische Handeln auf der Basis von geregelt wahrgenommener diagnostischer Information. Telemedizinisch unterstützte, strukturierte, vernetzte und meist intersektoral verankerte Versorgung (,Remote Patient Management ${ }^{\prime \prime}$ ) jenseits des reinen Monitorings ist die Voraussetzung für die Wirksamkeit telemedizinischer Konzepte. In weiteren randomisierten Studien [11, 12] wurde nachgewiesen, dass die Zeitspanne bis zur Wahrnehmung technischer und medizinischer Schwierigkeiten mithilfe der kontinuierlichen telemedizinischen Betreuung reduziert und somit einer drohenden Destabilisierung entgegengewirkt werden konnte. Im Gegensatz dazu zeigte die reine Übertragung der Daten keinen Mehrwert im Behandlungsprozess, wenn eine Handlungskonsequenz aus den übermittelten Daten ausblieb [13, 14].

\section{Rechtliche Rahmenbedin- gungen der telemedizinischen Versorgung}

Die Erprobung und Implementierung von telemedizinischen Diensten ziehen sowohl tatsächliche Veränderungen in den Behandlungsprozessen als auch rechtliche Herausforderungen nach sich. Hier sind insbesondere Unsicherheiten zu Haftungsrisiken aufseiten der Ärzteschaft zu benennen [15]. Das Bürgerliche Gesetzbuch und das Strafgesetzbuch stellen die Grundlagen für die Beantwortung dieser rechtlichen Fragestellungen im Rahmen der telemedizinischen Versorgung in Deutschland dar. Im Folgenden soll in Bezug auf die Telemonitoring-Nutzung insbesondere auf die Patientenaufklärung zu Mitwirkungsobliegenheiten, auf die Überwachungspflichten bei der Datenübermittlung und auf den Umgang mit Alarmmeldungen eingegangen werden [15].

Grundsätzlich können Haftungsfälle durch die Verletzung einer Pflicht aufgrund von Vorsatz oder Fahrlässigkeit ausgelöst werden. Es besteht daher zunächst aufseiten des Arztes eine Aufklärungspflicht mit vollumfänglicher Information des Patienten hinsichtlich der telemedizinischen Versorgung. Der Patient muss die ärztlichen Verhaltensempfehlungen verstanden haben und darüber aufgeklärt werden, dass er den Behandlungsprozess ggf. durch Mitwirkungshandlungen (z.B. Datentransfer) unterstützen muss [15].

Der Arzt ist im Rahmen einer telemonitorischen Versorgung dazu verpflichtet, die Funktionalität der Datenübermittlung der verwendeten telemetriefähigen Geräte in vorab definierten Zeitintervallen zu überwachen und bei ggf. auftretenden Mängeln in der Funktionsfähigkeit zu reagieren. Bei Nichtreaktion des Arztes kann ein Behandlungsfehler unterstellt werden [15].

Weitere rechtliche Fragestellungen beschäftigen sich mit den Reaktionszeiten und dem Umgang mit Alarmmeldungen durch den Arzt. Hierzu ist festzuhalten, dass Telemonitoring aufgrund von externer Sensorik oder implantierten telemetriefähigen Geräten in Deutschland nicht als Notfallsystem eingesetzt wird [2] und sich aufgrund der diskontinuierlichen Datenübertragung auch nicht als Notfallreaktionssystem eignet. Hingegen handelt es sich um eine datengestützte engmaschige diagnostische und therapeutische Begleitung gefährdeter Patienten mit kurzen, je nach auffälliger Befundkonstellation und Alarmmeldung vordefinierten Reaktionszeiten. Die zulässige Reaktionszeit des verantwortlichen Arztes im TMZ richtet sich nach der spezifischen Befundkonstellation. Er ist also nicht in jedem Fall zu einer sofortigen Reaktion beim Erhalt einer Alarmmeldung verpflichtet [15]. Im Rahmen des Aufklärungsgespräches sollte der Patient über diesen Sachverhalt vom Arzt mündlich und schriftlich informiert werden. Insbesondere ist hierbei klarzustellen, dass sich der Patient bei bestehenden akuten Beschwerden nicht auf die Meldung des Gerätes an den Arzt verlassen kann, sondern in ortsüblicher Weise das Rettungssystem alarmieren muss, um von diesem versorgt zu werden. Grundsätzlich bestehen derzeit keine festen Richtwerte zu den Reaktionszeiten des Arztes auf den Erhalt einer telemetrisch übertragenden Ereignismeldung. Jedoch sind Beispiele für typische Befundkonstellationen und vorgeschlagene Reaktionszeiten publiziert [4]. Im medizinischen Konsens wird zwar eine ärztliche Reaktion von $48 \mathrm{~h}$ als angemessen erachtet, jedoch gilt es hervorzuheben, dass der Arzt nach Gefährdungslage des 
Hier steht eine Anzeige.

黑 Springer 


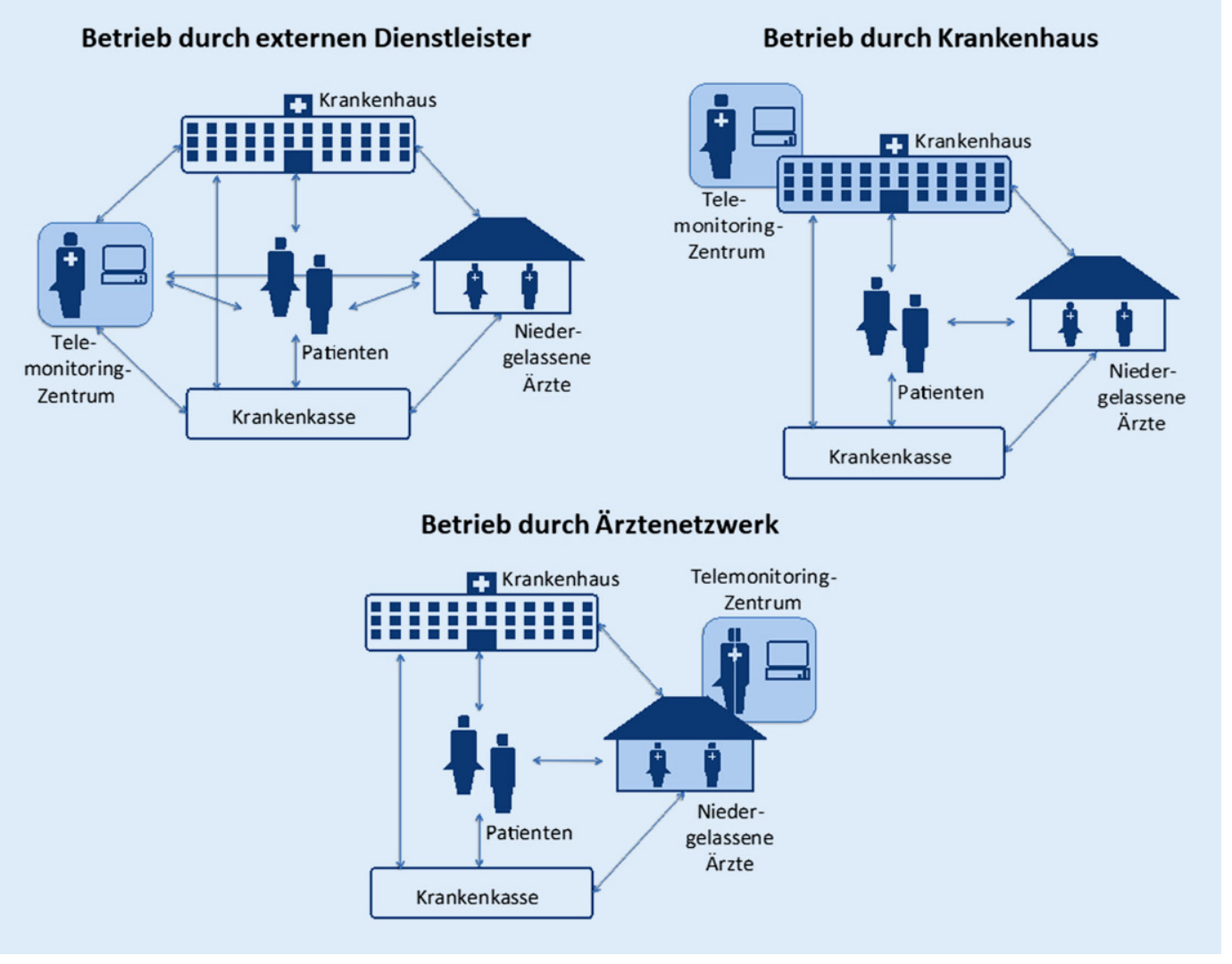

Abb. $2 \triangleleft$ Organisatorische Verankerungen eines TMZ. (Adaptiert nach [6, 16]; mit freundl. Genehmigung von (c) Georg Thieme Verlag KG. All rights reserved)

Patienten im Einzelfall entscheiden muss, wann eine Reaktion erforderlich ist. Sofern der verantwortliche Arzt eine Gefährdung des Patienten erkennt, besteht auch eine Handlungspflicht seitens des Arztes. Im Kontext der Haftungsbeschränkung ist eine telemedizinische Infrastruktur aufzubauen, die die Gefährdungslage des Patienten auch am Wochenende, nachts oder an Urlaubstagen überwacht und somit die Dringlichkeit der eingehenden Ereignismeldungen überprüft. Ist eine solche Infrastruktur gegeben, muss der Arzt auch entscheiden, ob unmittelbar oder innerhalb des Zeitraumes von $48 \mathrm{~h}$ reagiert werden muss [15]. Diese rechtlichen Rahmenbedingungen der telemedizinischen Versorgung müssen zu jedem Zeitpunkt im TMZ eingehalten werden.

\section{Organisatorische Verankerung der Telemedizinzentren}

Die organisatorische Verankerung und Ausgestaltung von telemonitorischen Diensten kann in unterschiedlicher Form in der Versorgungslandschaft realisiert werden [6]. So kann der Betrieb der tele- monitorischen Leistung z.B. durch einen externen Dienstleister, ein Krankenhaus, ein Ärztenetzwerk (allgemeine und/oder niedergelassene Ärzte) oder durch eine Krankenkasse bzw. Krankenversicherung erfolgen ([16], s. ๑ Abb. 2).

Bei externen Dienstleistern handelt es sich um juristisch und wirtschaftlich eigenständige Unternehmen, die nicht in die bestehenden Wettbewerbsstrukturen zwischen den medizinischen Leistungserbringern intervenieren [6]. In einer Kurzbefragung unter 54 Kardiologen wurden den extern verankerten TMZ die Gewährleistung der hohen Datenschutzanforderungen, die Abdeckung umfassender Servicezeiten sowie die ressourceneffiziente Erbringung der telemonitorischen Leistung durch eine hohe Standardisierung mehrheitlich zugesprochen [6].

Wird die telemonitorische Leistung durch ein TMZ angeboten, das an einem Krankenhaus verankert ist, so besteht eine fachliche und organisatorische Verbindung zwischen dem TMZ und dem Krankenhaus. Spezialisten können zudem zeitnah in den Behandlungsprozess eingebunden werden. Die befragten Ärzte hoben darüber hinaus eine mögliche Förderung des intra- und intersektoralen Austausches hervor, kritisierten jedoch auch, dass die Wettbewerbsneutralität zwischen ambulanten und stationären Strukturen nicht zwingend sichergestellt werden kann [6].

Ein Ärztenetzwerk stellt eine Vereinigung von niedergelassenen Haus- und/ oder Fachärzten dar [6]. Im Rahmen einer Kurzbefragung wurden durch die organisatorische Verankerung eines TMZ in einem Ärztenetzwerk eine Stärkung der bereits vorhandenen Arzt-PatientenBeziehung sowie die bestmögliche Förderung des intra- und intersektoralen Austausches erwartet. Kritik wurde in dieser Organisationsform an der Sicherstellung der Wettbewerbsneutralität geübt. Auch die Gewährleistung von umfassenden Servicezeiten wurde infrage gestellt [6].

\section{Warum ist die Zertifizierung von Telemedizinzentren notwendig?}

Um als integraler Bestandteil einer ganzheitlichen Patientenversorgung im Gesundheitssystem an Bedeutung zu gewin- 


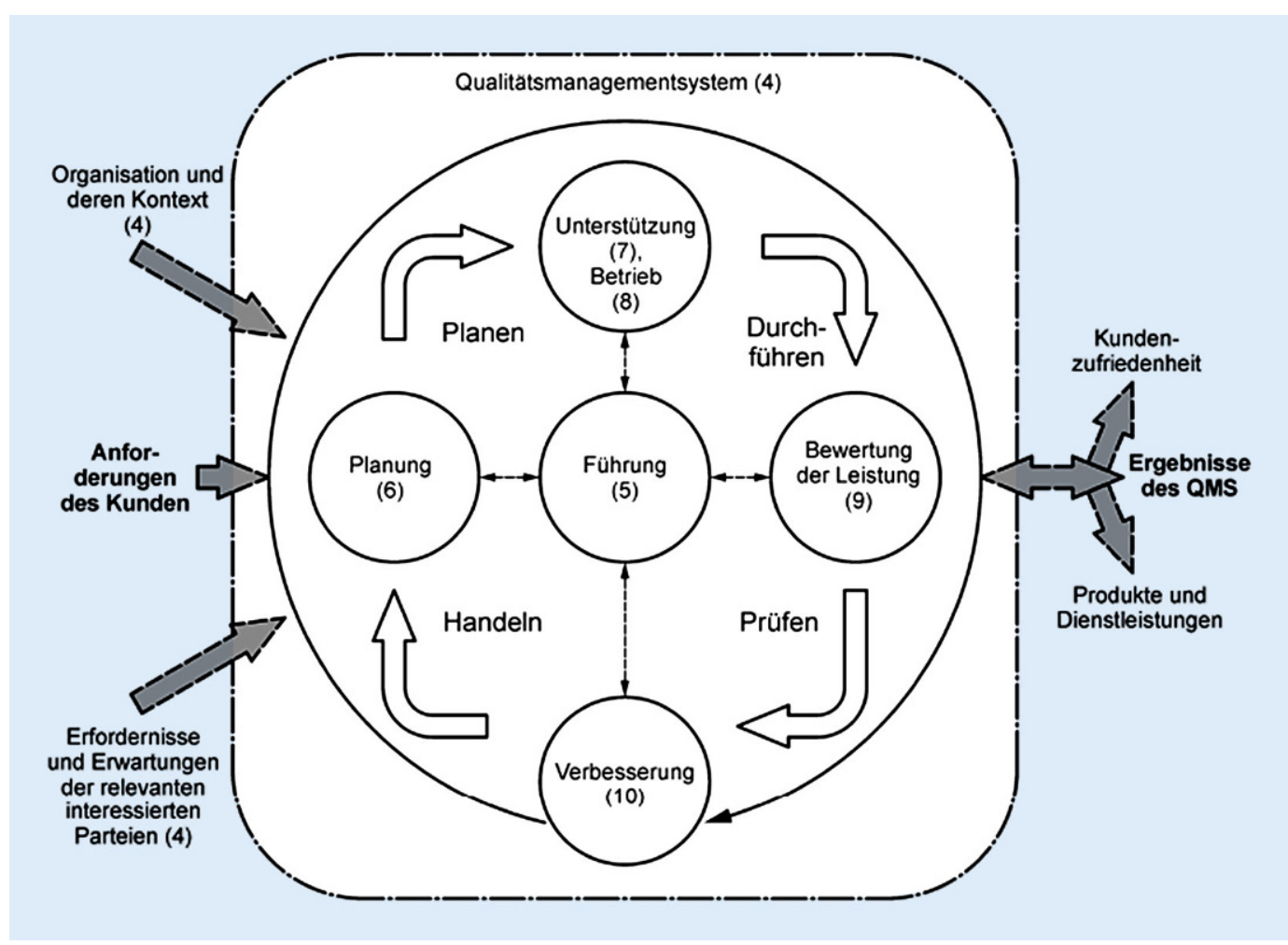

Abb. $3<$ Prozessmodell nach ISO 9001:2015 (DIN EN ISO 9001:2015, S. 13)

nen und telemedizinischen Dienstleistungen erfolgreich in die Regelversorgung zu verhelfen, müssen diese Einrichtungen neben einer einwandfreien technischen Funktionalität und Interoperabilität auch einheitliche Prozess- und Qualitätsstandards sicherstellen. Ziel ist es dabei, durch Definition struktureller und prozessualer Merkmale im Sinne überprüfbarer und nachvollziehbarer Qualitätskriterien der TMZ sicherzustellen, dass durch strukturierte Umsetzung von Telemonitoring und hierauf basierten strukturierten Behandlungsinterventionen die eingesetzten Mittel der Kostenträger im Gesundheitswesen eine tatsächliche Verbesserung der Versorgung herzkranker Patienten bewirken können.

Die Schaffung einheitlicher Prozessund Qualitätsstandards kann die Akzeptanz in der Bevölkerung steigern und so der Verbreitung von innovativen medizinischen Diensten dienen. Sie kann darüber hinaus einen Beitrag dazu leisten, bestehende Versorgungsunsicherheiten zu beseitigen [17]. Systematisches Qualitätsmanagement geht dabei über die Sicherung der Qualität einzelner Prozesse hinaus und zielt vielmehr auf die regelmäßige Prüfung, Verbesserung und
Weiterentwicklung der Prozesse sowie Verfahren in einer gesamten Einrichtung ab. Dadurch sollen die Versorgung der Patienten und auch die Motivation und Zufriedenheit der Mitarbeiter langfristig und kontinuierlich verbessert werden. Die umgesetzten Verbesserungen finden innerhalb eines konstanten Rahmens von Normen statt und haben daher einen inkrementellen Charakter. In diesem Zusammenhang wird auch von einem Singleloop-learning-Ansatz gesprochen, da die Effizienz der Handlungsabläufe in den Mittelpunkt gestellt wird [18].

Eine geeignete Möglichkeit, die Ausrichtung von Strukturen und Prozessen eines TMZ an gesetzlichen und normativen Anforderungen sicherzustellen, bietet die Zertifizierung des Qualitätsmanagementsystems [19].

In Deutschland sind nach § 135a SGB V bereits alle Leistungserbringer im System der gesetzlichen Krankenversicherung zur Sicherung und Weiterentwicklung der Qualität der von ihnen angebotenen Leistungen verpflichtet.

Im deutschen Gesundheitswesen empfehlen sich der Aufbau eines Qualitätsmanagementsystems gemäß DIN EN ISO 9001 und eine anschließende Bewertung und Zertifizierung durch „Benannte Stellen“, auch Notifizierte Stellen (Notified Bodies), der Europäischen Union. Es handelt sich hierbei um staatlich benannte und staatlich überwachte private Prüfstellen (Auditier- und Zertifizierstellen), die im Auftrag der Anbieter von Telemedizindienstleistungen tätig werden, um die Konformitätsbewertung von Herstellern von Industrieerzeugnissen und Dienstleistungen unterschiedlicher Art zu begleiten und zu kontrollieren. Sie üben damit „mittelbare Staatsverwaltung" aus. Hintergrund ist, dass in vielen Bereichen Produkte und Dienstleistungen in den Staaten der EU nur vertrieben und erbracht werden dürfen, wenn sie bestimmten (Sicherheits-)Anforderungen genügen. Im Fall der Zertifizierung von TMZ ist es zwingend notwendig, in Ergänzung zur Benannten Stelle die fachliche Expertise der DGK einzubeziehen.

Benannte Stellen sind z.B. die DQS$\mathrm{GmbH}$ (Deutsche Gesellschaft zur Zertifizierung von Managementsystemen), die KTQ-GmbH (Kooperation für Transparenz und Qualität im Gesundheitswesen) oder die Einrichtungen von TüV (Technische Überwachungsvereine). Benannte Stellen müssen und können nur gemeinsam mit 


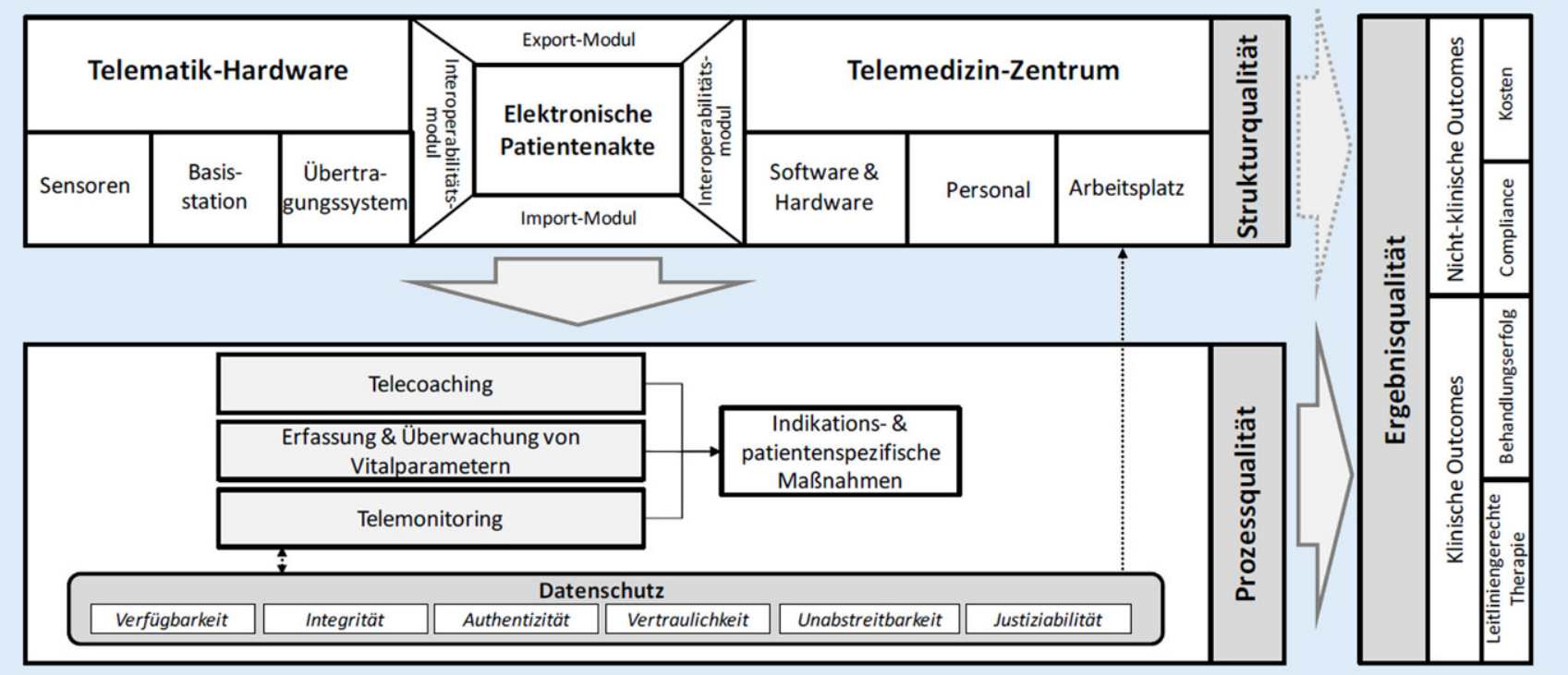

Abb. 4 \ Qualitätsdimensionen eines TMZ(eigene Darstellung)

der Deutschen Gesellschaft für Kardiologie - Herz- und Kreislaufforschung e.V. als medizinisch-inhaltlich überprüfende Fachorganisation und hier durch fachärztliche Mitglieder der spezialisierten Arbeitsgruppen die notwendige Qualitätsprüfung von $\mathrm{TMZ}$ in einem Zertifizierungsverfahren gewährleisten und sicherstellen.

Die verpflichtende Einführung eines Qualitätsmanagementsystems für TMZ muss zur Voraussetzung für die vergütete Erbringung von Leistungen des Telemonitorings gemacht werden, um eine Optimierung der vorhandenen Prozesse und eine Qualitätssicherung der telemedizinischen Versorgung zu gewährleisten [17].

Im Rahmen der international gültigen Norm DIN EN ISO 9001:2015 werden konkrete Anforderungen zum Aufbau und zur Weiterentwicklung eines Qualitätsmanagementsystems genannt [20], die eine gute Grundlage für die Zertifizierung einer Organisation bieten. Die DIN ISO 9001:2015 ist prozessorientiert aufgebaut und ermöglicht durch das Plan-Do-CheckAct-Modell (PDCA-Modell, s. - Abb. 3) und das risikobasierte Denken, dass die organisationseigenen Prozesse und Wechselwirkungen geplant werden können, um die Gesamtleistung der Organisation zu verbessern und zugleich Patientenzufriedenheit und Patientensicherheit zu erhöhen [20].
Die Implementierung und Zertifizierung eines Qualitätsmanagementsystems nach DIN EN ISO 9001:2015 bringen zudem Vorteile mit sich, die wie folgt auf das Setting des TMZ übertragen werden können [20]:

- Angebot von Produkten bzw. telemedizinischen Dienstleistungen, die die Patientenanforderungen sowie die hohen Anforderungen an die Patientenversorgung (gesetzliche, datenschutzrechtliche Anforderungen) erfüllen,

- Zeit- und Kosteneinsparung durch optimierte, standardisierte Prozesse,

- Erhöhung der Patientenzufriedenheit und Patientensicherheit,

- Behandlung von Risiken und Chancen im Rahmen der telemedizinischen Versorgung,

- dokumentierter Nachweis zur Konformität der festgelegten Anforderungen des Qualitätsmanagementsystems,

- Etablierung von standardisierten, qualitätsorientierten TMZ im deutschen Gesundheitswesen.

\section{Empfehlungen zur Zertifizierung von Telemedizinzentren}

Das Prozessmodell nach ISO 9001:2015 mit dem integrierten PDCA-Zyklus entspricht zudem der von Donabedian geprägten Unterscheidung in Struktur-, Prozess- und
Ergebnisqualität [21]. Im folgenden Abschnitt werden die grundlegenden Strukturmerkmale eines kardiologischen TMZ beschrieben, und dieser Systematik folgend werden die wesentlichen Qualitätsdimensionen in einem TMZ definiert. Die Qualitätsdimensionen in einem TMZ werden in - Abb. 4 dargestellt und sollten die Grundlage für eine Zertifizierung bilden.

\subsection{Strukturqualität}

Die Strukturqualität umfasst alle räumlichen, finanziellen und technischen Rahmenbedingungen und Voraussetzungen (sachliche Strukturqualität) sowie die fachlichen Fähigkeiten des Personals (personelle Strukturqualität). Eine Einrichtung, welche ein Qualitätsmanagementsystem nach ISO 9001:2015 eingeführt hat, muss demnach eine Führung haben, die sicherstellt, "dass die Anforderungen der Kunden [, Patienten und deren betreuende Ärzte,] und zutreffende gesetzliche sowie behördliche Anforderungen bestimmt, verstanden und beständig erfüllt werden" und "der Fokus auf die Verbesserung der Kundenzufriedenheit aufrechterhalten wird“ "[20]. Es gilt, die Qualitätsziele insbesondere im Hinblick auf die Betreuung und Behandlung der Patienten auszurichten und neben den medizinischen auch die technischen Kriterien im Rahmen der Qualitätspolitik zu definieren und zu dokumentieren. Konkret sind zu 
Hier steht eine Anzeige.

黑 Springer 


\section{Infobox}

Im Kontext der Struktur- und Prozessqualität bilden somit folgende Aspekte die Grundlage und unabdingbare Voraussetzung für die durch die DGK zu zertifizierenden Telemedizinzentren

- Es muss zwingend die Sicherstellung eines 24/7/365-Facharztstandards gewährleistet werden.

- Die Dienstplaneinsicht muss nachvollziehbar dargestellt werden.

- Sollte ein TMZ durch niedergelassene Ärzte/niedergelassene Ärztenetzwerke betrieben werden, muss eine Sicherstellung der Datenbewertung außerhalb der Officezeiten, durch Aufschalten an ein durch die DGK zertifiziertes überregionales TMZ gewährleistet werden.

- Es müssen eine überprüfbare, detaillierte Darstellung der Alarmübermittlungen und -quittierungen (TMZ $\rightarrow$ PBA $\rightarrow T M Z$ ) gemäß G-BA-Beschluss und eine plausible Darstellung der Prozesse in der elektronischen Plattform/FA vorgehalten werden und einsehbar sein.

- Es wird als die Aufgabe des TMZ betreibenden Arztes verstanden, ausschließlich ordnungsgemäß zertifizierte Produkte einzusetzen, (auch) um die erforderliche Behandlungsqualität zu gewährleisten.

- Um ein TMZ qualitätsgesichert betreiben zu können, bedarf es ferner einer Zertifizierung dessen als gesamte Einheit. Eine solche Zertifizierung kann auch durch den Hersteller der (gesamten) Plattform erfolgt sein, sollte hingegen ein TMZ durch Komponenten unterschiedlicher Plattformhersteller "zusammengestellt" worden sein, so ist diese Einheit gesondert (entsprechend der DIN ISO 9001) zu zertifizieren.

- Die beschriebenen Anforderungen im Rahmen der Struktur- und Prozessqualität (z. B. Drittsoftware, Datenschutz, Managementabläufe) erfordern das genannte Vorgehen im Zertifizierungsprozess. Im Hinblick auf den zu erwartenden Wettbewerb sei die Einbindung einer akkreditierten Stelle sinnvoll und erforderlich.

- Zudem muss die Einhaltung der technischen und organisatorischen Maßnahmen (TOM) nach § 32 der DatenschutzGrundverordnung (DS-GVO) inklusive der Sicherstellung des Data-Hostings in Deutschland gewährleistet sein.

- Es ist als Grundlage ein Qualitätsmanagement-Handbuch vorzuhalten, in dem alle medizinischen Prozesse und Grenzwertüberschreitungen verschriftlich sind inklusive der notwendigen SOPs. fordernde Strukturmerkmale für ein TMZ bereits in einer früheren Publikation vorgeschlagen worden [2].

Systemanforderungen. Ein wesentliches Merkmal der Strukturqualität in einem TMZ stellt die Infrastruktur dar, die das eigentliche TMZ, Software, Hardware sowie Informations- und Kommunikationstechnik umfasst. Innerhalb des TMZ müssen internetfähige Computerarbeitsplätze mit einer international freigeschalteten Telefonanlage zur Verfügung stehen [2]. Für die Hardwareumgebung ist dabei nicht nur die Qualität der Geräte zur Messung und Übertragung von definierten physiologischen Vitalparametern der Patienten essenziell, sondern auch die einfache Handhabung durch die Patienten, die ohne technische Vorkenntnisse möglich sein muss. Die Diagnose-Hardware befindet sich entweder integriert in ein Implantat oder als externe Sensorik, jeweils mit geeignetem Datentransmitter, bei dem Patienten vor Ort. Zentrale Software-Komponenten sind bei implantatbasiertem Monitoring die Telemonitoringplattformen der Gerätehersteller und nach Möglichkeit direkte Datenübertragungsschnittstellen zu der elektronischen Plattform. Die daraus verfügbar gemachte datenbasierte Patienteninformation und daraus abgeleitete Interventionen werden in der elektronischen Plattform als weiterer zentraler Softwarekomponente dokumentiert. In der elektronischen Plattform als Kernelement des TMZ werden die persönlichen Daten des Patienten, alle für die Krankheit relevanten anamnestischen Parameter sowie Informationen über die Funktionalität der Übertragung, auffällige Befundkonstellationen, Alarme und Interventionen reproduzierbar gespeichert.Ziel ist es, dass alle an der Patientenversorgung beteiligten Akteure - unter Berücksichtigung der jeweiligen Zugriffsrechte einrichtungsübergreifend Möglichkeit der Einsichtnahme zur Dokumentation haben. Der Austausch von medizinisch relevanten Daten des TMZ mit den an der Behandlung beteiligten Arztpraxen, Krankenhäusern und Rehabilitationskliniken ist sicherzustellen. Perspektivisch sollte die elektronische Plattform eines TMZ über datenschutzkonforme Schnittstellen zur direkten Übermittlung von relevanten
Diagnose- und Behandlungsdaten verfügen. Angesichts der Tatsache, dass die Implementierung einer einrichtungsübergreifenden Patientenakte an der derzeit noch eingeschränkten Interoperabilität der Primärsysteme scheitert, mit denen die verschiedenen Einrichtungen arbeiten, kommt der Kommunikationsfähigkeit der elektronischen Plattform im TMZ mit internen und externen Partnern als ein erster Schritt auf dem Weg zu einer einrichtungsübergreifenden elektronischen Plattform daher eine besondere Bedeutung zu. Um eine möglichst große Interoperabilität aller Software-Komponenten realisieren zu können, müssen den Schnittstellen offene Standards (z. B. XML, Java, XML-RPC) zugrunde liegen [5]. Auf Basis des Gesetzes für sichere digitale Kommunikation und Anwendungen im Gesundheitswesen (E-Health-Gesetz) soll zudem die Telematik-Infrastruktur gefördert und eine einheitliche Telematik-Plattform genutzt werden, die den sicheren Austausch der sensiblen Gesundheitsdaten der Patienten erleichtert [3].

\section{Raumbedarf und technische Ausstat-} tung. Neben der Software und der Hardware zählen der Raumbedarf und die technische Ausstattung des Telemedizinischen Zentrums zu wichtigen Parametern der Strukturqualität. Hierbei ist insbesondere darauf zu achten, dass die Arbeitsumgebung so gestaltet ist, dass die datenschutzrechtlichen Vorschriften eingehalten werden. Voraussetzungen hierfür bilden u. a. Zugangs-, Zugriffs- und Zutrittskontrollen sowie entsprechend geschützte Backoffice Arbeitsplatzsysteme und Datenträger.

Personal. Die notwendigen personellen Ressourcen zur Realisierung der telemedizinischen Dienstleistung und zur Einführung und Aufrechterhaltung des Qualitätsmanagementsystems müssen vorhanden sein. Die Leitung des kardiologischen TMZ muss durch einen Facharzt für Innere Medizin und Kardiologie erfolgen, der in Abhängigkeit der zu behandelnden Krankheitsentitäten über die Zusatzqualifikation „Spezielle Rhythmologie/aktive Herzrhythmusimplantate" und/ oder "Herzinsuffizienz" der DGK verfügen muss [2]. Zudem sollte wenigstens ein 
weiterer ärztlicher Mitarbeiter, der in der Telekardiologie geschult ist, im TMZ arbeiten. Das nichtärztliche Personal, welches die telemedizinischen Dienstleistungen ausführt oder unterstützt, muss über eine angemessene medizinische Ausbildung verfügen (z.B. Gesundheits- und Krankenpfleger, medizinische Fachangestellte, medizinisch-technische Angestellte) und eine dokumentierte Einweisung für die Anwendung relevanter Hard- und SoftwareKomponenten erhalten. Zusätzlich zur medizinischen Ausbildung sind indikationsspezifische Schulungen zur Herzinsuffizienz (orientiert am Curriculum der European Society of Cardiology für die Heart Failure Nurse [22] bzw. Fortbildungscurriculum: spezialisierte Herzinsuffizienz-Assistenz [23] und zur Therapie mit Herzrhythmusimplantaten, - Herzschrittmacher-, Defibrillator- und Resynchronisationstherapie, Diagnostik mit implantierbaren kardialen Monitoren/Ereignisrekordern) [2] oder vergleichbare Qualifikationen, erforderlich. Inhalt von Schulungen muss angesichts der vielfältigen geforderten Kommunikationsaufgaben jenseits der sorgfältigen Analyse von eingehenden Vitalparametern auch die Erfordernis empathischer, professioneller und wertschätzender Kommunikation mit den chronisch kranken Patienten und den Kooperationspartnern sein $[2,24]$. Das TMZ kann, basierend auf den genannten Inhalten, qualifizierende Schulungen anbieten [5]. Eine ausführliche Erläuterung zu den Schulungsinhalten ist bei Helms et al. [2] nachzulesen. Des Weiteren müssen auch für die an der Behandlung des Patienten angeschlossenen verantwortlichen Ärzte entsprechende indikationsspezifische Fortbildungsmodule angeboten werden, die mit einer CMEZertifizierung akkreditiert werden müssen [5]. Die ärztlichen Mitarbeiter des TMZ müssen in einem Zeitraum von 2 Jahren insgesamt 20 Fortbildungspunkte aus kardiologischer Fortbildung erlangen [2].

Prozessumgebung. Nach ISO 9001:2015 zählt auch die Prozessumgebung zu den erforderlichen Ressourcen. Sie soll die Motivation und Leistung der agierenden Mitarbeiter positiv beeinflussen, die so wiederum zur Patientenzufriedenheit und zum Unternehmenserfolg beitragen. Hierzu zählen u.a. eine angenehme Ar- beitsatmosphäre, ergonomische Arbeitsbedingungen, Sicherheitsbestimmungen sowie Hygienestandards [20].

\subsection{Prozessqualität}

Telemonitoring und -coaching. Die Prozessqualität bezieht sich grundsätzlich auf alle Aktivitäten, die im Laufe der Patientenversorgung im TMZ getroffen werden. Im Zentrum des eigentlichen telemedizinischen Versorgungsprozesses stehen die Überwachung und Begutachtung der eingehenden Vitalparameter (Telemonitoring) sowie die telefonische strukturierte Betreuung von Patienten durch medizinisch ausgebildetes Fachpersonal (Telecoaching). Letzteres kann unterschieden werden in das Annehmen von eingehenden Telefonanrufen (Inbound) sowie die proaktive telefonische Kontaktaufnahme mit dem Patienten (Outbound). Insbesondere bei den (optionalen) planmäßigen, vom TMZ ausgehenden Anrufen, bei denen der Patient in standardisierter Form zu Lebensqualität, Medikation, klinischer Symptomatik und zur Häufigkeit von Arztbesuchen und Klinikaufenthalten befragt werden kann, stehen dabei eine Verhaltensmodifikation, eine gesteigerte Adhärenz sowie die Schulung und Sensibilisierung des Patienten als wesentliche Ziele im Vordergrund. Die regelmäßige Patientenschulung gemäß den zu prüfenden protokollbezogenen Schulungskonzepten findet durch strukturierte telefonische Gespräche statt und kann durch E-Learning-Module ergänzt werden. Innerhalb der Schulungseinheiten soll sowohl der Umgang mit den telemetriefähigen Geräten geprobt werden als auch eine Edukation zur eigenen kardiologischen Erkrankung und zum Umgang mit der Erkrankung erfolgen, um langfristig die Selbstwirksamkeit und Adhärenz des Patienten zu erhöhen [4]. Darüber hinaus hat der Patient selbst auch die Möglichkeit, sich bei technischen, organisatorischen oder medizinischen Fragen telefonisch 24/7/365 ans TMZ zu wenden.

Standardarbeitsabläufe und Dokumentation. Der Aufbau eines Referenzsystems ist für die klare Beurteilung der Patienten durch die ärztlichen Mitarbeiter unerlässlich. Dabei sollten sich die eindeutig strukturierten und diagnostischen Vorgehensweisen und Checklisten an den aktuellen indikationsspezifischen Leitlinien orientieren [5]. Die abgeleiteten Handlungskonsequenzen müssen transparent in der elektronischen Plattform des TMZ und in Übereinstimmung mit den "Standard Operation Procedures" (SOPs) und den aktuell geltenden Datenschutzbestimmungen dokumentiert werden [2]. Die Datenschutzbestimmungen müssen dabei in allen Prozessen des TMZ berücksichtigt werden und umfassen die Aspekte Verfügbarkeit, Vertraulichkeit, Authentizität, Integrität, Unabstreitbarkeit sowie Justiziabilität.

\subsection{Ergebnisqualität}

Die Ergebnisqualität der Begleitung gefährdeter Patienten durch Telemonitoring wird durch die Struktur- und Prozessqualität mit den oben angeführten Merkmalen bedingt. Die Ergebnisqualität kann an der Realisation evidenzbasierter, leitliniengerechter Therapie und am stabilen oder verbesserten Gesundheitszustand eines Patienten gemessen werden. Eine weitere Dimension der Ergebnisqualität ist die Patientenzufriedenheit. Daneben stellt auch die Adhärenz des Patienten eine weitere wichtige nichtklinische Qualitätsmaße dar. Nach DIN ISO 9001:2015 muss das TMZ die Konformität der telemedizinischen Dienstleistungen sicherstellen und die Wirksamkeit des Qualitätsmanagementsystems kontinuierlich verbessern. Entsprechende Dokumentations- und Analyseprozesse zur wiederkehrenden überwachenden Qualitätskontrolle müssen implementiert werden [20]. Hierzu gehören neben der Messung der Patientenzufriedenheit auch regelmäßig durchzuführende Audits (s. Abschn. 8.4) und Prüfungen der telemedizinischen Dienstleistungen. Weitere Instrumente der Qualitätssicherung, die der Steigerung der Leistungsfähigkeit des TMZ dienen, können eine krankheitsspezifische Versorgungsregisterteilnahme, Organisation von Qualitätszirkeln sowie ein dokumentiertes Verfahren zum Umgang mit Fehlern sein.

Der Anspruch auf eine ganzheitliche, versorgungseinrichtungsübergreifende Ausrichtung eines telemedizinischen Zentrums geht mit zahlreichen Anforderungen 


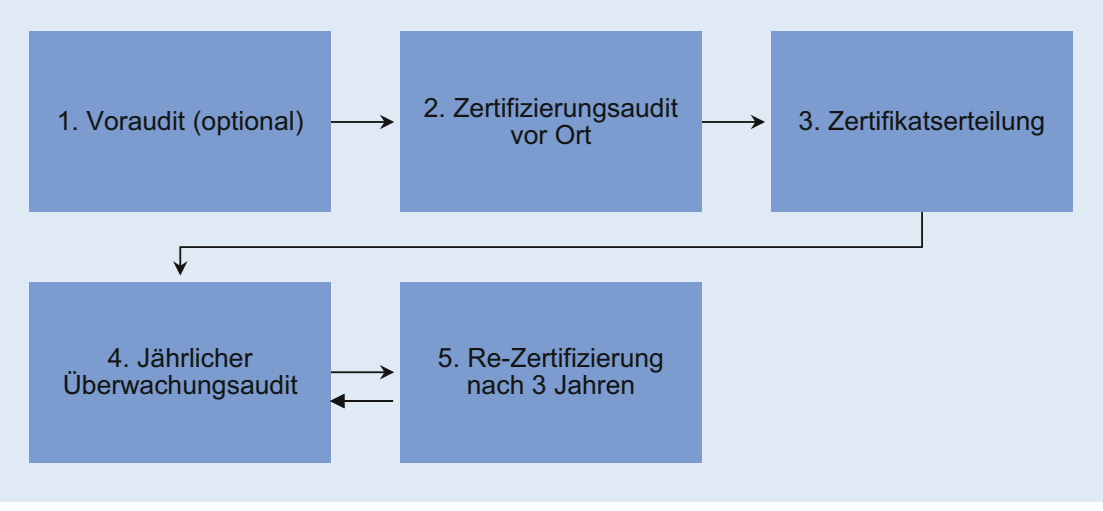

Abb. 5 ム Zertifizierungsprozess eines TMZ nach DIN EN ISO 9001:2015. (Adaptiert nach [24])

an die Qualität in den Strukturen, den Prozessen und schließlich den Ergebnissen einher. Vor diesem Hintergrund ist ein Qualitätsmanagementsystem unverzichtbar, welches sicherstellt, dass die Qualität der Prozesse, Strukturen und Ergebnisse geprüft und optimiert wird mit dem Ziel einer dauerhaften Verbesserung der telemedizinischen Versorgung von Patienten mit kardiologischen Erkrankungen sowie ihrer Zufriedenheit. Eine Zertifizierung ermöglicht darüber hinaus eine objektive Überprüfung des Qualitätsmanagementsystems und eine gezielte Ausrichtung des TMZ an den gesetzlichen und normativen Anforderungen.

\subsection{Der Zertifizierungsprozess}

Die Zertifizierung des Qualitätsmanagementsystems eines TMZ muss von einer unabhängigen, externen Institution durchgeführt werden, die mit den Zertifizierungsprozessen vertraut ist. Derzeit wird im Gesundheitswesen eine Zertifizierung von verschiedenen Zertifizierungsgesellschaften auf dem Markt angeboten (z.B. DQS-GmbH, KTQ-GmbH, TÜV Rheinland). Das TMZ sollte dabei selbst entscheiden, durch wen die Zertifizierung erfolgt. Grundsätzlich muss in diesem Zusammenhang die Rolle der DGK durch die med.wiss. Fachexpertise, insbesondere durch die von der DGK benannten Experten abgebildet werden. Die DGK als Fachgesellschaft spricht sich auf Basis der in diesem Positionspapier genannten Qualitätsanforderungen an ein TMZ für eine zukünftige Zertifizierung gemeinsam mit einer benannten Stelle aus. Es ist zwingend notwendig, das Expertenwissen der
DGK im Rahmen der Zertifizierungsprozesse zu nutzen und die medizinischen Umsetzungen zu überprüfen.

Der Zertifizierungsprozess für ein Qualitätsmanagementsystem nach DIN EN ISO 9001:2015 variiert je nach Serviceleistungen der aktuellen Anbieter leicht, bleibt in seiner Grundstruktur jedoch einheitlich (s. - Abb. 5). Im Folgenden wird der für das TMZ mögliche Zertifizierungsprozess nach DIN EN ISO 9001:2015 kurz skizziert. Nach dem ersten Informationsgespräch mit den Verantwortlichen des TMZ zum Prozessablauf findet ein optionales Voraudit (1) statt [25]. Dabei handelt es sich um eine vor dem eigentlichen Prüfprozess stattfindende Vorbeurteilung zur generellen Umsetzung der Normanforderungen. Im zweiten Schritt erfolgt das Zertifizierungsaudit (2) vor Ort im TMZ. Hierbei handelt es sich um ein Systemaudit, welches die Wirksamkeit des Qualitätsmanagementsystems des gesamten TMZ analysiert und auf Schwachstellen, Fehlerursachen und Verbesserungspotenziale überprüft. Im Zuge dessen werden die Beschreibungen und die dokumentierten Prozesse des Qualitätsmanagementsystems (z. B. SOPs, Checklisten, Qualitätsmanagementhandbuch) durch die Auditoren kontrolliert, und ggf. wird die praktische Anwendung demonstriert. Das Qualitätsmanagementsystem wird von den Auditoren in einem Auditbericht abschließend bewertet. Bei einem positiven Bescheid erhält das TMZ einen Nachweis für die erfolgreiche Zertifizierung. Das Zertifikat (3) bescheinigt die Normenkonformität und Funktionsfähigkeit des Qualitätsmanagementsystems des TMZ. Gleichzeitig bedeutet dies für die Mitarbeitenden des
TMZ, dass das erfolgreich geprüfte Qualitätsmanagementsystem tagtäglich "gelebt" werden muss. Um einen kontinuierlichen Verbesserungsprozess des Qualitätsmanagementsystems zu etablieren, sollten weiterhin jährliche Überwachungsaudits (4) zur Bewertung der Praxisumsetzung des Qualitätsmanagementsystems, Prozessoptimierung sowie Normkonformität stattfinden. Eine Re-Zertifizierung (5) sollte 3 Jahre nach Erstzertifizierung erfolgen. Dabei werden die Schritte 2 bis 5 in der - Abb. 5 wiederholt, und das TMZ könnte somit die Zertifizierung seines Qualitätsmanagementsystems für weitere 3 Jahre verlängern [25].

\section{Schlussfolgerungen und Ausblick}

Durch die verschiedenen Strukturmerkmale eines TMZ müssen Versorgungs-, Steuerungs-, Monitoring- und Kommunikationsprozesse in einem TMZ integriert werden, die die Einführung eines systematischen Qualitätsmanagements erfordern. Mithilfe der geforderten Zertifizierung des eingeführten Qualitätsmanagementsystems werden die Struktur-, Prozessund Ergebnisqualität der telemonitorischen Dienstleistungen objektiv geprüft und die Konformität mit den gesetzlichen Verordnungen und Anforderungen öffentlichkeitswirksam bestätigt. Dies kann zu einer systematischen Verbesserung des TMZ im Gesundheitswesen führen, da das Vertrauen der Patienten sowie der medizinischen Partner in die telemedizinischen Leistungen der zertifizierten Einrichtungen gestärkt wurde. Die einheitlich festgelegten Qualifizierungskriterien für das telemedizinisch tätige Personal, die eine Grundlage für eine leitliniengerechte und sektorenübergreifende Betreuung der Patienten sowie eine qualifizierte Personalisierung bieten, fördern zudem die Investitionsbereitschaft der deutschen Medizintechnik und steigern die Akzep$\operatorname{tanz}$ der Kostenträger. Darüber hinaus sollten die Prozessabläufe in einem TMZ zukünftig auch verstärkt durch Anwendungen der künstlichen Intelligenz (KI) unterstützt werden, z. B. bei der Stratifizierung von Patienten, bei der frühzeitigen Erkennung einer Gesundheitsverschlechterung oder einer frühzeitigen und beschleunigten Entscheidungsfindung [26]. Somit 
könnten Diagnose- und Behandlungsfehler vermieden sowie die Entwicklung einer personalisierten Therapie integriert werden.

\section{Alternative Qualitätsmanagement- systeme}

Die DGK behält sich unter der Maxime größtmöglicher Qualität vor, in Zukunft neben der DIN ISO-Zertifizierung weitere Qualitätsstandards (Qualitätsmanagementnormen) zuzulassen, wenn diese in gleicher Weise geeignet sind, die DGKdeterminierten Qualitätskriterien für die Zertifizierung von TMZ abzubilden.

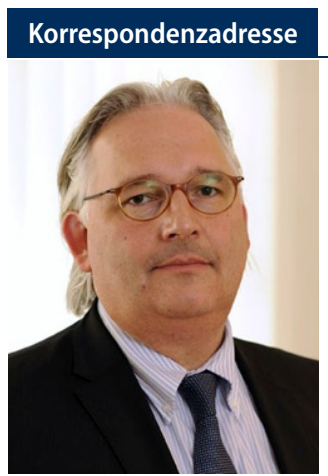

Dr. Thomas M. Helms

Research, Peri Cor Arbeitsgruppe Kardiologie Scharpenbargshöhe 10D, 21149 Hamburg, Deutschland

thomas_helms@t-online.de

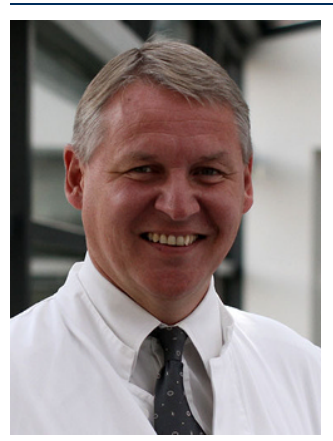

\section{Prof. Dr. Christian A. Perings}

Kath. Klinikum Lünen-Werne, Med. Klinik I, Kardiologie, Pneumologie und Intensivmedizin, St.-Marien-Hospital Lünen

Lünen, Deutschland

perings.christian@klinikum-luenen.de

\section{Einhaltung ethischer Richtlinien}

Interessenkonflikt. Den Interessenkonflikt der Autoren finden Sie online auf der DGK-Homepage unter http://leitlinien.dgk.org/bei der entsprechenden Publikation.

\section{Position paper on certification of telemedicine centers. Recommendations of the working group 33 telemonitoring in the German Cardiac Society (DGK)}

Cardiovascular diseases, especially chronic heart failure, are associated with increased morbidity and mortality. In the context of patient care, telemedical services have great potential to address the growing imbalance between the need for treatment and current provision of care. Cardiological telemedicine centers (TMCs) can thus support patient care in rural or remote areas and enable fast access to specialists. To ensure uniform process and quality standards, the implementation of a systematic quality management system in TMCs is essential. The predefined verifiable and comprehensible quality criteria of a TMC are intended to ensure that the structured implementation of telemonitoring and structured treatment interventions based on it can bring about an actual improvement in the care of patients with heart disease. In addition, certification of the quality management system ensures that the structures and processes of a TMC are aligned with legal and normative requirements.

\section{Keywords}

Telemedicine · Telemonitoring · Quality management · Certification · Quality of care

Für diesen Beitrag wurden von den Autoren keine Studien an Menschen oder Tieren durchgeführt. Für die aufgeführten Studien gelten die jeweils dort angegebenen ethischen Richtlinien.

\section{Literatur}

1. Gemeinsamer Bundesausschuss (30. März 2021) Beschluss des Gemeinsamen Bundesausschusses über eine Änderung der Richtlinie Methoden vertragsärztliche Versorgung: Telemonitoring bei Herzinsuffizienz. BAnz AT

2. Helms TM, Stockburger M, Köhler F et al (2019) Grundlegende Strukturmerkmale eines kardiologischen Telemedizinzentrums für Patienten mit Herzinsuffizienz und implantierten Devices, Herzrhythmusstörungen und erhöhtem Risiko für den plötzlichen Herztod. Empfehlungen der Arbeitsgruppe 33 Telemonitoring in der Deutschen Gesellschaft für Kardiologie, Herz- und Kreislaufforschung e.V. Herzschr Elektrophys 30:136-142

3. Helms TM, Stockburger $M$, Köhler $F$ et al (2019) Positionspapier Telemonitoring. Von den Nukleusmitgliedern der AG33 Telemonitoring in der DGK und assoziierten Mitgliedern. Herzschr Elektrophys 30:287-297

4. Helms TM, Stockburger M, Schwab JO et al (2019) Befundkonstellation und abgeleitete Behandlungsinterventionen bei telemedizinisch überwachten Patienten mit Herzinsuffizienz, Herzrhythmusstörungen oder erhöhtem Risiko für den plötzlichen Herztod. Herzschr Elektrophys 30:298-305

5. Helms TM, Müller A, Perings C et al (2017) Das Telemedizinische Zentrum als essenzieller Baustein konzeptioneller Ansätze zum Telemonitoring kardialer Patienten. Herzschr Elektrophys 28:293-302

6. Augustin U, Zippel-Schultz B, Schwab JO et al (2016) Organisatorische Verankerung und Ausgestaltung von Telemonitoring-Zentren für die Betreuung herzinsuffizienter Patienten aus Sicht der medizinischen Leistungserbringer. Aktuel Kardiol 5:129-134

7. Mcmurray JJV, Stewart S (2002) The burden of heart failure. Eur Heart J Suppl 4:D50-D58

8. Köhler F, Prescher S, Köhler K (2019) Telemedizin bei Herzinsuffizienz. Internist 60:331-338

9. Köhler F, Köhler K, Deckwart O et al (2018) Efficacy of telemedical interventional management in patients with heart failure (TIM-HF2): a randomised, controlled, parallel-group, unmasked trial. Lancet 392:1047-1057

10. Hindricks G, Taborsky M, Glikson M et al (2014) Implant-based multiparameter telemonitoring of patients with heart failure (IN-TIME): A randomised controlled trial. Lancet 384:583-590

11. Crossley GH, Boyle A, Vitense $\mathrm{H}$ et al (2011) The CONNECT (clinical evaluation of remote notification to reduce time to clinical decision) trial: the value of wireless remote monitoring with automatic clinician alerts. J Am Coll Cardiol 57:1181-1189

12. Varma N, Epstein AE, Irimpen A et al (2010) Efficacy and safety of automatic remote monitoring for implantable cardioverter-defibrillator follow-up: the Lumos-T safely reduces routine office device follow-up (TRUST) trial. Circulation 122:325-332

13. Morgan JM, Kitt S, Gill J et al (2017) Remote management of heart failure using implantable electronic devices. Eur Heart J 38:2352-2360

14. Stockburger M, Helms TM, Perings CA et al (2017) Nutzenbewertung des strukturierten Telemonitorings mithilfe von aktiven Herzrhythmusimplantaten. Kardiologe 11:452-459

15. Schneider H (2017) Rechtliche Rahmenbedingungen der telemedizinischen Versorgung. Herzschr Elektrophys 28:303-306

16. Viktor V, Dittmar R, Nagel E (2011) Positionierung eines Telemedizinsichen Zentrums. In: Duesberg F (Hrsg) e-Health 2011 Informationstechnologien und Telematik im Gesundheitswesen. medical future, Solingen, S 223-227

17. Benner MJ (2009) Dynamic or static capabilities? Process management practices and response to technological change. J Prod Innov Manag 26:473-486 


\section{Positionspapiere}

18. ArgyrisC, SchönDA (1978)Organizationallearning: A theory of action perspective. Addison-Wesley, Reading

19. Farberow $B$, Hatton $V$, Leenknecht $C$ et al (2008) Caveat emptor: The need for evidence, regulation, and certification of home telehealth systems for the management of chronic conditions. Am J Med Qual 23:208-214

20. Deutsches Institut Für Normung E.V. (2015) Qualitätsmanagementsysteme - Anforderungen (ISO 9001:2015). Beuth, Berlin, S71

21. Donabedian A (1980) Explorations in quality assessment and monitoring: The definition of quality and approaches to its assessment. Health Administration Press, Ann Arbor

22. Riley JP, Astin F, Crespo-Leiro MG et al (2016) Heart Failure Association of the European Society of Cardiology heart failure nurse curriculum. Eur $J$ Heart Fail 18:736-743

23. Störk S, Kindermann I, Jacobs Met al (2020) Fortbildungscurriculum: Spezialisierte HerzinsuffizienzAssistenz. Aktuel Kardiol 9:90-95

24. Budych K, Pelleter J, Schultz C et al (2010) Qualifikationskonzept Telemedizin-Assistent - Professionalisierung der Telemedizin. E-HealthCOM 4:54-56

25. TÜV Rheinland (2021) ISO 9001 Zertifizierung Qualitätsmanagementsystem

26. Zippel-Schultz B, Schultz C, Müller-Wieland D et al (2021) Künstliche Intelligenz in der Kardiologie. Relevanz, aktuelle Anwendungen und nächste Schritte. Herzschr Elektrophys 201(32):89-98. https://doi.org/10.1007/s00399-020-00735-2

MED UPDATE SEMINARE 2022

Cardio Update 2022

17. DGK-Kardiologie-Update-Seminar

04.-05. März 2022

Mainz und Livestream

11.-12. März 2022

Berlin und Livestream

Wiss. Leitung:

Prof. Dr. Michael Böhm, Homburg

Prof. Dr. Stephan Achenbach, Erlangen

Prof. Dr. Ulrich Laufs, Leipzig

Prof. Dr. Thorsten Lewalter, München

Unter der Schirmherrschaft der DGK, DGIM

www.cardio-update.com

Auskunft für alle Update-Seminare: med update $\mathrm{GmbH}$

www.med-update.com

Tel.: 0611 - 736580

info@med-update.com 